\title{
Synthesis method for amorphous metallic foam
}

\author{
Jan Schroers ${ }^{\text {a) }}$ \\ Keck Laboratory, California Institute of Technology, Pasadena, California 91125 \\ and Liquidmetal Technologies, Lake Forest, California 92630
}

Chris Veazey, Marios D. Demetriou, and William L. Johnson

Keck Laboratory, California Institute of Technology, Pasadena, California 91125

(Received 23 June 2004; accepted 21 September 2004)

\begin{abstract}
A synthesis method for the production of amorphous metallic foam is introduced. This method utilizes the thermodynamic stability and thermoplastic formability of the supercooled liquid state to produce low-density amorphous metallic foams in dimensions that are not limited to the critical casting thickness. The method consists of three stages: the prefoaming stage, in which a large number of small bubbles are created in the equilibrium liquid under pressure; the quenching stage, in which the liquid prefoam is quenched to its amorphous state; the foam expansion stage, in which the amorphous prefoam is reheated to the supercooled liquid region and is processed under pressures substantially lower than those applied in the prefoaming step. Results from a dynamic model suggest that the foam expansion process is feasible, as the kinetics of bubble expansion in the supercooled liquid region are faster than the kinetics of crystallization. Within the proposed synthesis method, bulk amorphous foam products characterized by bubble volume fractions of as high as 85\% are successfully produced. (C) 2004 American Institute of Physics.
\end{abstract}

[DOI: $10.1063 / 1.1818355]$

\section{INTRODUCTION}

Metallic foams find increasingly more interest as structural materials. This is to a large extent due to high stiffness in conjunction with very low specific weight and high energy-absorption capability. ${ }^{1}$ Foams can be classified as either open or closed porous. Open foams are characterized by interconnected bubbles and are mainly used as functional materials. Closed foams are characterized by spatially separated bubbles and find applications as structural materials. It has been found that perfection of foam morphology has a crucial influence on its mechanical properties. ${ }^{2}$

Since foam is an unstable structure, the process of foaming of pure metals is a challenging task. The dynamics of bubble nucleation, growth, sedimentation, merge, and collapse scale inversely with viscosity. The viscosity of a pure metallic liquid at its melting temperature is of the order of $10^{-3} \mathrm{~Pa} \mathrm{~s}^{3}$ The viscosity of a bulk metallic glass (BMG)forming liquid at the liquidus temperature is of the order of $1 \mathrm{Pas}^{4}$ Therefore, the foaming kinetics of BMGs are expected to be drastically slower compared to pure metals. In order to accomplish such sluggish foaming kinetics in aluminum, experiments were performed in space under microgravity conditions. ${ }^{5}$ The sluggish foaming kinetics of BMGforming liquids could enable better control over bubble size distribution, spatial homogeneity, and bubble volume fraction. Such controllability over foam morphology is expected to yield foams that exhibit superior mechanical properties.

The challenge in vitrifying is circumventing crystallization upon quenching. A minimum quench rate is required to bypass crystallization, which is termed the critical cooling

\footnotetext{
${ }^{a)}$ Author to whom correspondence should be addressed; Electronic mail: jan.schroers@liquidmetal.com
}

rate. The quench rate is dictated by the rate of heat removal such that limitations are imposed on the dimensions of products that can be cast amorphous. The largest size that can be cast amorphous is often identified as the critical casting thickness. Recently, an amorphous metallic foam product was successfully produced by a method involving bubble generation and expansion above the liquidus temperature and subsequent quenching to the amorphous state. ${ }^{6}$ This method is illustrated schematically in Fig. 1. The drawback of this method is that the foam dimensions are further limited by the critical cooling rate of the alloy, as a consequence of a dramatic reduction in the "effective" thermal diffusivity of the foamed material due to the presence of gas bubbles. Therefore, the critical cooling rate imposes an upper bound on the dimensions of amorphous foam products, as well as a lower bound on foam density, and hence limits their potential applicability. Thus, techniques in which bubbles are created

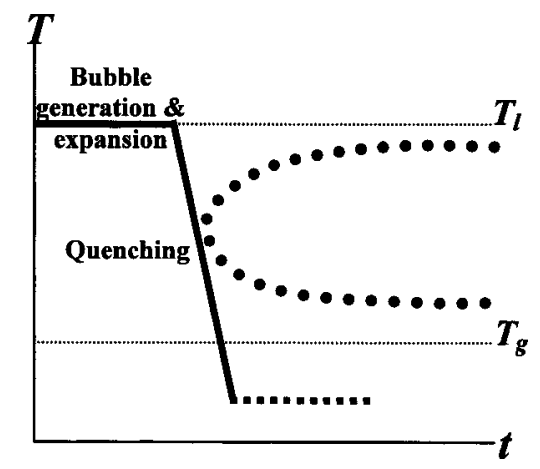

FIG. 1. Foaming method by bubble creation and expansion in the liquid state and subsequent quench of the foamed liquid to its amorphous state. Symbols $T_{l}$ and $T_{g}$ denote liquidus and glass transition temperatures, respectively. 
and expanded in the equilibrium liquid are not very promising for commercial production of bulk amorphous foams.

In this study, a foam-synthesis method is introduced that utilizes the thermodynamic stability and thermoplastic formability of the supercooled liquid state. In this method therefore, the constraints dictated by the critical cooling rate are removed such that low-density amorphous foams in dimensions that are not limited to the critical casting thickness can be produced. The fundamentals of this method are outlined in the following section. The effects of the viscosity characteristics of BMG-forming liquids on the morphology of the final foam product are also discussed in Sec. II. In Sec. III, the feasibility of processing foam expansion in the supercooled liquid region is assessed by means of a dynamic model that simulates the bubble expansion kinetics in a BMG-forming liquid. In Sec. IV, the details of the experimental method are outlined, and the amorphous prefoam and foam products developed by this method are presented.

\section{FOAM-PROCESSING ABILITY OF BMGS}

One of the most unique features of BMG-forming liquids is the existence of a supercooled liquid state that is continuous and exhibits excellent stability against crystallization. This metastable liquid state appears in a temperature region between glass transition and crystallization. This state can be reached by rapidly quenching from the equilibrium liquid state, however, it can be attained more conveniently by reheating from the amorphous state. Viscosity in this region varies smoothly between $10^{12} \mathrm{~Pa}$ s and $10^{7} \mathrm{~Pa} \mathrm{~s}$, and crystallization is extremely sluggish. Consequently processing windows for thermoplastic forming of the liquid can be utilized in this region. Owing to the high viscosities characterizing the supercooled liquid, the kinetics of foaming would be extremely sluggish as well. Furthermore the strong dependence of viscosity on temperature would allow for precise control of the foam expansion process by varying temperature within the supercooled region.

The unique characteristics of the supercooled liquid state can be utilized to develop a three-step manufacturing method for producing amorphous metallic foams. The first step involves the generation of a large number of small bubbles into the equilibrium liquid under pressure to produce a prefoam having a small bubble volume fraction. The intermediate step involves quenching of the bubbly liquid to its amorphous state by cooling at rates greater than the critical cooling rate. The last step involves reheating of the sample to the supercooled liquid region and reducing the pressure to values substantially lower than those used in the prefoaming step in order to activate bubble expansion. This method is illustrated schematically in Fig. 2. Since in this method bubble expansion takes place below the nose of the temperature-timetransformation (TTT) diagram, the critical cooling rate to bypass crystallization is essentially infinite. Therefore it becomes trivial to quench the foam from the supercooled liquid state to the amorphous state. Cooling-rate constraints on vitrification are therefore relaxed and consequently low-density amorphous foams in dimensions that are not limited to the critical casting thickness can be produced by this method.

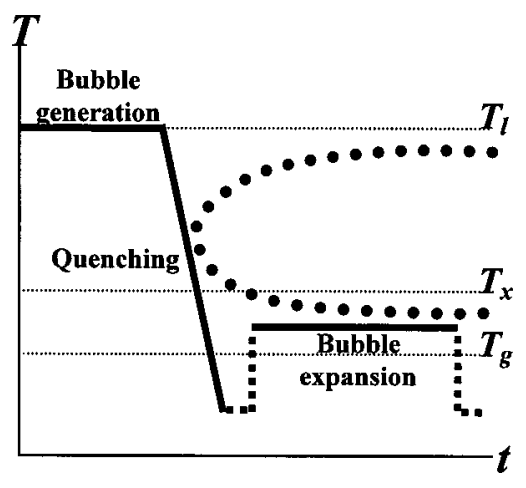

FIG. 2. Proposed three-step foaming method: (1) a large number of small bubbles are created in the equilibrium liquid under pressure; (2) the liquid prefoam is quenched to its amorphous state; (3) the amorphous prefoam is reheated to the supercooled liquid region and the pressure is substantially reduced to activate bubble expansion. Symbols $T_{l}, T_{x}$, and $T_{g}$ denote liquidus, crystallization, and glass transition temperatures, respectively.

Owing to the excessively high viscosities characterizing BMG-forming liquids, bubble sedimentation can be reduced to negligible levels. The sedimentation velocity $u$ of a gas bubble in a liquid can be approximated by Stokes equation:

$$
u=\frac{8 R^{2}\left(\rho_{l}-\rho_{g}\right) g}{9 \eta},
$$

where $R$ is the radius of the bubble; $\rho_{l}$ and $\rho_{g}$ are the densities of liquid and gas, respectively; $g$ the gravitational acceleration, and $\eta$ is the viscosity. To evaluate the sedimentation dynamics in BMG-forming liquids, the sedimentation velocity of 1 atm argon gas bubbles in the $\mathrm{Pd}_{43} \mathrm{Ni}_{10} \mathrm{Cu}_{27} \mathrm{P}_{20}$ liquid is considered at two different temperatures: $870 \mathrm{~K}$ and $650 \mathrm{~K}$. The temperature of $870 \mathrm{~K}$ is the liquidus temperature of this alloy, while the temperature of $650 \mathrm{~K}$ is within the supercooled liquid region. Therefore these temperatures represent the processing temperatures for the prefoaming stage (step 1) and the foam expansion stage (step 3), respectively. Ideal-gas law is used for the density of argon, while for $\mathrm{Pd}_{43} \mathrm{Ni}_{10} \mathrm{Cu}_{27} \mathrm{P}_{20}$ liquid the density is taken as $9340 \mathrm{~kg} / \mathrm{m}^{3}$ (measured) and the viscosities at $870 \mathrm{~K}$ and $650 \mathrm{~K}$ are taken as $0.6 \mathrm{~Pa} \mathrm{~s}$ and $1.1 \times 10^{6} \mathrm{~Pa} \mathrm{~s}$, respectively. ${ }^{7} \mathrm{~A}$ limit for detectable sedimentation can be established by considering that in $1 \mathrm{~cm}$ samples, sedimentations of less than $1 \mathrm{~mm}$ during experimental times of $100 \mathrm{~s}$ are undetectable. This criterion therefore suggests that undetectable stratification can be characterized by sedimentation velocities of less than $10 \mu \mathrm{m} / \mathrm{s}$. The results for the sedimentation velocity as a function of bubble radius as estimated from Eq. (1) are shown in Fig. 3. At the temperature of $870 \mathrm{~K}$, where prefoam processing takes place, the criterion for negligible sedimentation can be fulfilled for bubble radii of less than $10 \mu \mathrm{m}$. This suggests that sedimentation would be undetectable in prefoams with an average bubble size of $10 \mu \mathrm{m}$. At the temperature of $650 \mathrm{~K}$, however, where foam expansion is processed, the criterion for negligible sedimentation can be fulfilled for bubble radii of less than $1 \mathrm{~cm}$. This implies that during foam expansion, bubble sizes in the centimeter range can be produced without detectable sedimentation.

Therefore owing to the excessively high viscosities of BMG-forming liquids, no density gradients along the gravity 


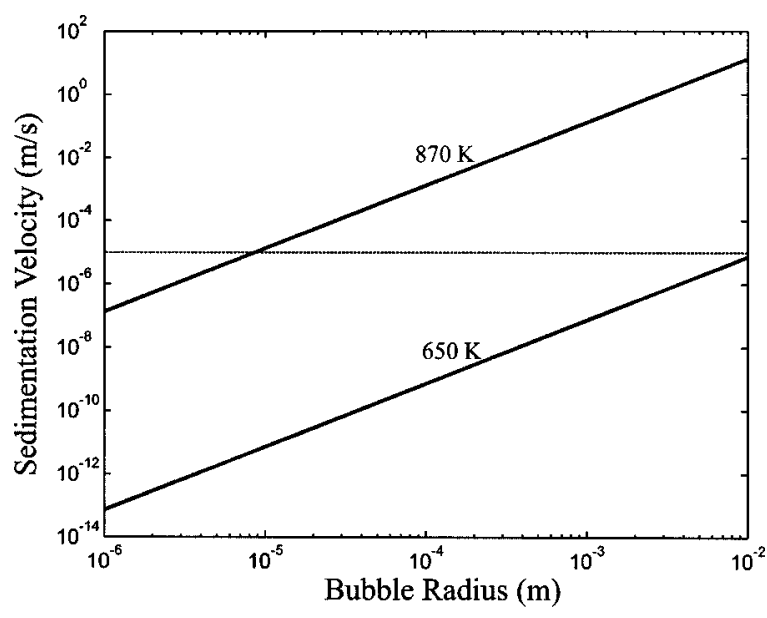

FIG. 3. The sedimentation velocity of bubbles in $\mathrm{Pd}_{43} \mathrm{Ni}_{10} \mathrm{Cu}_{27} \mathrm{P}_{20}$ liquid as a function of bubble radius at temperatures $870 \mathrm{~K}$ (liquidus) and $650 \mathrm{~K}$ (deeply undercooled region) as computed by Eq. (1).

direction caused by bubble sedimentation are expected to form in foam products produced by this method. However, nonuniformities in the morphologies may appear in foams produced by this method. Such nonuniformities are attributed to the strong dependence of viscosity on temperature in the supercooled liquid region. As will be demonstrated in the following section, the kinetics of bubble expansion scale inversely with viscosity. Therefore, even shallow temperature gradients during foam expansion can give rise to steep viscosity gradients, which would contribute to spatially nonuniform bubble growth and ultimately to nonuniform morphology. Therefore in order to achieve spatially uniform foam morphologies by this method, it is essential to process foam expansion under uniform temperatures. The foams developed in this preliminary stage were expanded using either compact-resistance heater or rf-coil, neither of which produces uniform temperature distributions. Therefore the morphologies of the foam products developed in this study, which are presented in Sec. IV, appear to be only moderately uniform. The primary intent of the current study, however, is to demonstrate the potential of the proposed synthesis method to produce low-density amorphous foams that are not limited to the critical casting thickness. Work is underway to develop a furnace apparatus by which foam expansion can take place under uniform temperatures. Such isothermal annealing conditions are expected to substantially improve the uniformity in foam morphology.

\section{FOAM-EXPANSION KINETICS IN BMG-FORMING LIQUIDS}

The bubbles created in the amorphous prefoam ingot have attained an equilibrium size, which is dictated by the ambient pressure used during the prefoaming process. In order to activate bubble expansion, the ambient pressure must be lowered such that a pressure difference between gas and liquid is imposed, hence constituting a driving force for expansion. In order to kinetically enable bubble expansion, the prefoam must be reheated into the supercooled liquid region such that a reduction in viscosity is effected. Heating into the supercooled liquid region however, would also enhance the kinetics of crystallization. Consequently the time allowed for expansion would be limited. Therefore, the processing temperature for expansion has to be carefully determined by considering the simultaneous kinetics of bubble expansion and crystallization. An ideal processing window can arise when the time scale characterizing bubble expansion is shorter than that characterizing crystallization. In this section, a model for the kinetics of foam expansion in a BMGforming liquid is established in order to assess the feasibility of the foam expansion process. The model utilizes available kinetic data for the supercooled liquid and evaluates the expansion kinetics of individual bubbles by assuming the bubbles to maintain spherical symmetry and the liquid to be infinite and incompressible. The current model is not intended to accurately simulate the expansion evolution during foaming, but to rather give an approximate assessment of the feasibility of processing foam expansion in the supercooled liquid region.

Denoting the bubble pressure on the gas side of the interface as $p_{i}$, the pressure on the liquid side of the interface as $p_{o}$, and the far-stream liquid pressure as $p_{\infty}$, the total driving force for growth $\left(p_{i}-p_{\infty}\right)$ can be expressed as a sum of an interfacial driving force $\left(p_{i}-p_{o}\right)$ and a far-stream driving force $\left(p_{o}-p_{\infty}\right)$, as follows:

$$
\left(p_{i}-p_{\infty}\right)=\left(p_{i}-p_{o}\right)+\left(p_{o}-p_{\infty}\right) .
$$

The interfacial kinetics are taken as suggested by Poritsky: ${ }^{8}$

$$
\left(p_{i}-p_{o}\right)=4 \eta \frac{d R / d t}{R}+\frac{2 \sigma}{R},
$$

where $\sigma$ is the surface tension, $\eta$ is the liquid viscosity, and $R$ is the time-dependent bubble radius. The first term on the right represents the principal normal stresses on the bubble while the second term constitutes the surface tension contribution. The far-stream inertia kinetics are given by the irrotational-flow analysis of Rayleigh ${ }^{9}$ as follows:

$$
\left(p_{o}-p_{\infty}\right)=\rho\left[R \frac{d^{2} R}{d t^{2}}+\frac{3}{2}\left(\frac{d R}{d t}\right)^{2}\right],
$$

where $\rho$ is the liquid density. Furthermore, the pressure at the gas side of the bubble interface can be related to the number of moles of the gas $n$ by assuming ideal gas law:

$$
p_{i}=\frac{n \mathcal{R} T}{(4 \pi / 3) R^{3}}
$$

where $\mathcal{R}$ is the gas constant and $T$ is temperature. The number of moles $n$, which is conserved during expansion, can be obtained from knowledge of the initial gas pressure in the bubble $p_{0}$. Substituting Eqs. (3)-(5) into Eq. (2) we obtain

$$
\rho R \frac{d^{2} R}{d t^{2}}+\frac{3}{2} \rho\left(\frac{d R}{d t}\right)^{2}+\frac{4 \eta}{R} \frac{d R}{d t}=\frac{n \mathcal{R} T}{(4 \pi / 3) R^{3}}-\frac{2 \sigma}{R}-p_{\infty} .
$$

Equation (6) is a differential equation governing the growth evolution of a single bubble from an initial size $R_{0}$ to an equilibrium size $R_{e q}$, which can be obtained by setting $d R / d t=d^{2} R / d t^{2}=0$ in Eq. (6). Owing to the excessively high viscosities characterizing BMG-forming liquids, Eq. (6) can be simplified substantially by means of a scaling analysis. 
Two relaxation processes take place during bubble growth: far-stream hydrodynamic relaxation, which for such irrotational flow is characterized by a time scale $\tau_{\text {hyd }}=\Delta R \sqrt{\rho / \Delta p}$, and interfacial stress relaxation, which is characterized by a diffusive time scale $\tau_{v i s}=\rho \Delta R^{2} / 4 \eta$. In the above expressions $\Delta R=R_{e q}-R_{0}$ and $\Delta p=p_{0}-p_{\infty}$. Defining dimensionless variables as $\hat{R}=R / \Delta R$ and $\hat{t}=t / \tau_{\text {hyd }}$, Eq. (6) can be scaled as follows:

$$
\begin{aligned}
\hat{R} \frac{d^{2} \hat{R}}{d \hat{t}^{2}}+\frac{3}{2}\left(\frac{d \hat{R}}{d \hat{t}}\right)^{2}+\frac{\tau_{\text {hyd }}}{\tau_{\text {vis }}} \frac{1}{\hat{R}} \frac{d \hat{R}}{d \hat{t}} \\
\quad=\frac{n \mathcal{R} T}{\Delta p(4 \pi / 3) \Delta R^{3}} \frac{1}{\hat{R}^{3}}-\frac{2 \sigma}{\Delta p \Delta R} \frac{1}{\hat{R}}-\frac{p_{\infty}}{\Delta p} .
\end{aligned}
$$

For excessively high viscosities the time-scale ratio becomes $\tau_{\text {hyd }} / \tau_{\text {vis }} \gg 1$ such that the inertia contribution [first two terms on the left of Eq. (6)] becomes vanishingly small compared to the interfacial stress contribution (third term on the left). Accordingly the evolution equation can be simplified to the following dimensional form:

$$
\frac{4 \eta}{R} \frac{d R}{d t}=\frac{n \mathcal{R} T}{(4 \pi / 3) R^{3}}-\frac{2 \sigma}{R}-p_{\infty} .
$$

Equation (8) can be used to model the growth evolution of a single bubble expanding in a highly viscous liquid. Since inertia terms are vanishingly small, the constraint for the liquid to extend to infinity can therefore be relaxed. This implies that interactions between adjacently expanding bubbles would be negligible, i.e., bubbles would essentially expand like balloons. The above argument, however, is only true when no merging of bubbles takes place. Nevertheless under the assumption of no bubble merging, Eq. (8) could be employed to adequately simulate the growth evolution of a size distribution of bubbles expanding in a viscous liquid.

In the context of this study, the supercooled liquid will be assumed to be structurally relaxed to its equilibrium state during expansion, i.e., its viscosity will be assumed to be at its Newtonian value. Under this assumption, nonequilibrium effects contributed by quenched-in free volume or induced by strain rates during growth are neglected. In practice, however, such nonequilibrium effects may become important and may contribute to dramatically decrease the viscosity and hence substantially increase the kinetics of expansion. Under such assumption, therefore, the kinetics are accounted for conservatively and hence expansion is evaluated at the slowest possible rate. The Newtonian viscosity of $\mathrm{Pd}_{43} \mathrm{Ni}_{10} \mathrm{Cu}_{27} \mathrm{P}_{20}$ as evaluated recently by Fan et al. ${ }^{7}$ will be employed: $\quad \log _{10}(\eta)=-6.8+2 \times 2880 /\left\{T-447+\left[(T-447)^{2}\right.\right.$ $\left.+4 \times 7.3 T]^{1 / 2}\right\}$ Pa s. The surface tension of $\mathrm{Pd}_{43} \mathrm{Ni}_{10} \mathrm{Cu}_{27} \mathrm{P}_{20}$ can be approximated by that of $\mathrm{Pd}_{76} \mathrm{Cu}_{6} \mathrm{Si}_{18}{ }^{10} \sigma=1.399$ $+0.26 \times 10^{-3}(T-1033) \mathrm{N} / \mathrm{m}$. For the density of $\mathrm{Pd}_{43} \mathrm{Ni}_{10} \mathrm{Cu}_{27} \mathrm{P}_{20}$, the measured value of $\rho=9340 \mathrm{~kg} / \mathrm{m}^{3}$ will be utilized.

In order to evaluate the accuracy of Eqs. (6) and (8), and hence validate the scaling analysis given by Eq. (7), the expansion of a $100 \mu \mathrm{m}$ bubble in supercooled $\mathrm{Pd}_{43} \mathrm{Ni}_{10} \mathrm{Cu}_{27} \mathrm{P}_{20}$ liquid at $650 \mathrm{~K}$ for $3000 \mathrm{~s}$ is considered. The initial bubble pressure $p_{0}$ is taken to be $10^{5} \mathrm{~Pa}$, while the far-stream liquid

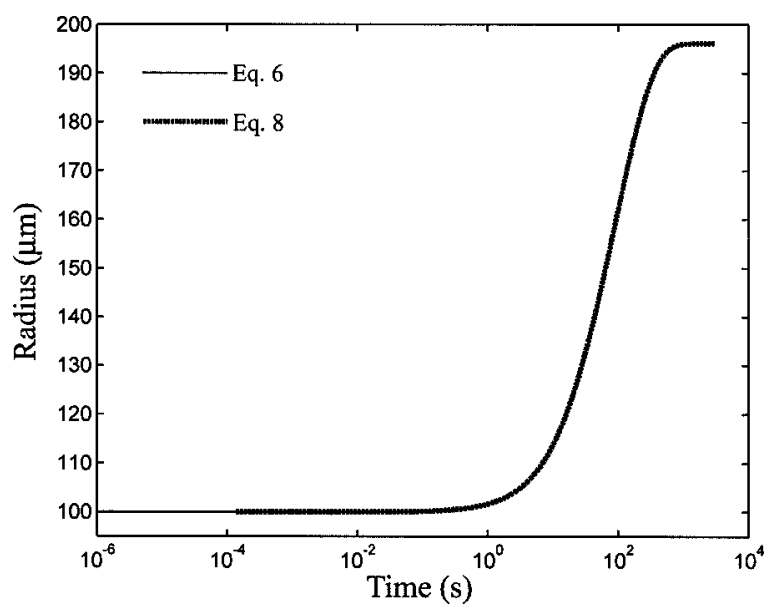

FIG. 4. Expansion evolution of a $100 \mu \mathrm{m}$ radius bubble initially at $p_{i}$ $=10^{5} \mathrm{~Pa}$ expanding in undercooled $\mathrm{Pd}_{43} \mathrm{Ni}_{10} \mathrm{Cu}_{27} \mathrm{P}_{20}$ liquid at $675 \mathrm{~K}$ and $p_{\infty}=1 \mathrm{~Pa}$, as computed by Eqs. (6) and (8).

pressure $p_{\infty}$ is taken to be $1 \mathrm{~Pa}$. Under these conditions, the time-scale ratio becomes $\tau_{\text {hyd }} / \tau_{\text {vis }} \sim 10^{5}$, hence implying negligible inertia effects, and accordingly Eq. (8) must be a good approximation to Eq. (6). Equations (6) and (8) and are both nonlinear and were therefore solved numerically. A MATLAB solver with a relative tolerance of $10^{-12}$ was employed. The computed evolutions from the initial size to the equilibrium one predicted by the two equations are shown in Fig. 4. The evolutions appear to be identical, hence verifying the validity of the scaling analysis and suggesting that Eq. (8) is adequate to simulate evolution in a highly viscous liquid.

The developed model, Eq. (8), is employed to investigate the effect of varying temperature in the supercooled liquid region on the expansion evolution. Expansion of a $100 \mu \mathrm{m}$ bubble for $3000 \mathrm{~s}$ in undercooled $\mathrm{Pd}_{43} \mathrm{Ni}_{10} \mathrm{Cu}_{27} \mathrm{P}_{20}$ liquid was considered. The far-stream pressure was taken to be $1 \mathrm{~Pa}$. The computed evolutions at temperatures 625,650 , and $675 \mathrm{~K}$ for an initial gas pressure of $10^{5} \mathrm{~Pa}$ are shown in Fig. 5. The results suggest that for the processing conditions considered, the bubbles at $650 \mathrm{~K}$ and $675 \mathrm{~K}$ would attain the

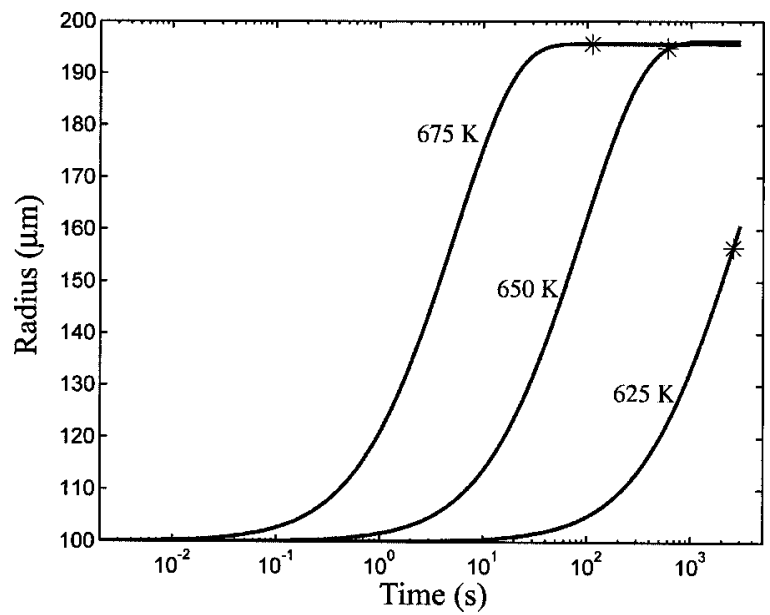

FIG. 5. Expansion evolution of a $100 \mu \mathrm{m}$ radius bubble initially at $p_{i}$ $=10^{5} \mathrm{~Pa}$ expanding in undercooled $\mathrm{Pd}_{43} \mathrm{Ni}_{10} \mathrm{Cu}_{27} \mathrm{P}_{20}$ liquid at $p_{\infty}=1 \mathrm{~Pa}$ and temperatures of $625 \mathrm{~K}, 650 \mathrm{~K}$, and $675 \mathrm{~K}$. The onset of crystallization at each temperature is marked with an asterisk. 


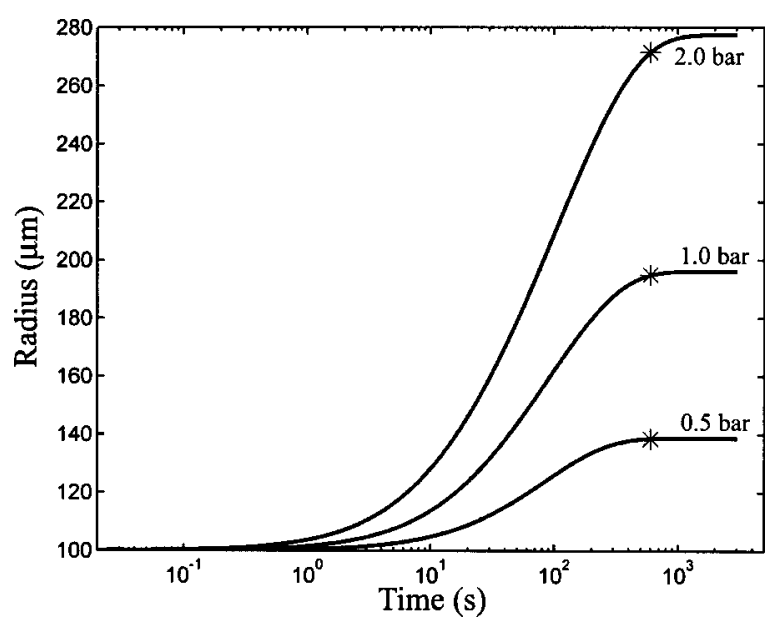

FIG. 6. Expansion evolution of a $100 \mu \mathrm{m}$ radius bubble initially at pressures of $0.5 \times 10^{5}, 1.0 \times 10^{5}$, and $2 \times 10^{5} \mathrm{~Pa}$ expanding in undercooled $\mathrm{Pd}_{43} \mathrm{Ni}_{10} \mathrm{Cu}_{27} \mathrm{P}_{20}$ liquid at $650 \mathrm{~K}$ and $p_{\infty}=1 \mathrm{~Pa}$. The onset of crystallization at $650 \mathrm{~K}$ is marked with an asterisk.

equilibrium size, while the one at $625 \mathrm{~K}$ would grow substantially but would not attain equilibrium. From the results it appears that the expansion kinetics are enhanced substantially by increasing temperature as a consequence of the dramatic decrease in viscosity. The viscosities at temperatures 625,650 , and $675 \mathrm{~K}$ are $3.5 \times 10^{7}, 1.1 \times 10^{6}$, and 6.6 $\times 10^{4} \mathrm{~Pa}$ s, respectively. In order to assess whether adequate expansion is possible before crystallization sets in, the expansion kinetics must be compared against crystallization kinetics. This can be accomplished by superimposing the time for isothermal crystallization, as obtained from an experimental TTT diagram, onto the expansion evolution. The crystallization times at temperatures 625,650 , and $675 \mathrm{~K}$ are obtained from the experimental TTT diagram ${ }^{11}$ as 2470,600 , and $112 \mathrm{~s}$, respectively, and are superimposed on the plot by an asterisk. From the results it appears that at $675 \mathrm{~K}$ and $650 \mathrm{~K}$, expansion equilibrates before crystallization sets in, however, at $625 \mathrm{~K}$ crystallization precedes equilibration. The model therefore confirms that under the slowest possible kinetic conditions (i.e., equilibrium Newtonian conditions), expansion in the deeply undercooled liquid is feasible, as a processing time window exists between expansion and crystallization.

Additionally, the model is utilized to explore the effect of initial gas pressure on bubble expansion. The same conditions as above were used. The computed evolutions for initial gas pressures of $0.5 \times 10^{5}, 1.0 \times 10^{5}$, and 2.0 $\times 10^{5} \mathrm{~Pa}$ at temperature $650 \mathrm{~K}$ are shown in Fig. 6. As expected, higher initial gas pressure results in greater equilibrium bubble size, since it corresponds to greater molar quantities. The expansion kinetics at different pressures appear similar: relaxation to equilibrium size occurs between $300-500 \mathrm{~s}$ in all three cases. This is attributed to the fact that the dependence of kinetics on molar quantity is very weak as compared to the dependence on viscosity. The time for the onset of crystallization at $650 \mathrm{~K}$ is also superimposed on the plot, suggesting that similar processing time windows exists at all pressures.

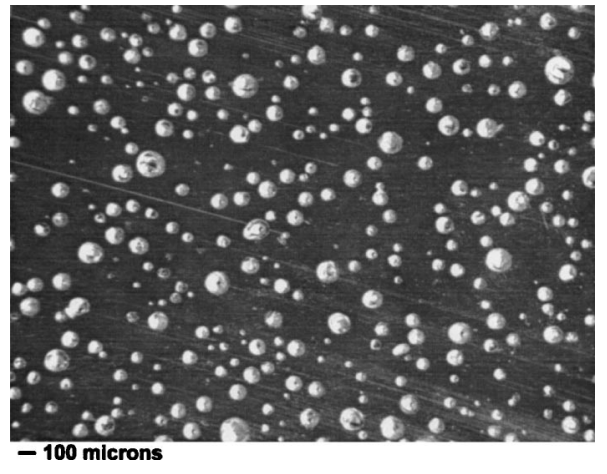

FIG. 7. $\mathrm{Pd}_{43} \mathrm{Ni}_{10} \mathrm{Cu}_{27} \mathrm{P}_{20}$ prefoam processed for 5 min at 2 bar pressure and $1170 \mathrm{~K}$. It consists of $15 \mathrm{vol} \%$ bubbles homogeneously distributed throughout the prefoam with an average bubble size of $70 \mu \mathrm{m}$.

\section{EXPERIMENTAL PRODUCTION OF AMORPHOUS METAL FOAM}

In this section, the experimental methods employed for the preparation of the prefoam and for the expansion of the foam are outlined. Furthermore, the amorphous prefoam and foam products developed by implementing the method proposed in this study are presented.

\section{A. Prefoam preparation}

During the prefoaming process, a large number of small bubbles are introduced into the liquid sample. The morphology of a final foam product should exhibit narrow bubble size distribution as well as uniform spatial distribution of bubbles. It is therefore critical that the prefoam morphology also possesses those size and spatial distribution characteristics, so that it could evolve into a desirable foam product. Amorphous metal prefoams can be generally produced by introduction of bubbles in the equilibrium liquid under pressure, and by subsequent quench of the liquid to its amorphous state. In order to attain cooling rates high enough to bypass crystallization, prefoams should additionally possess small bubble volume fractions such that the effective thermal properties are not extensively degraded. In this study, prefoam production was achieved by means of two techniques: gas-releasing agents and mechanical air entrapment.

In the gas-releasing agent technique, a requirement for the agent is to have low reactivity with the BMG-forming alloy, as a reaction could degrade its glass-forming ability. Most importantly, however, the agents must be able to release gas at temperatures near the liquidus temperature of the alloy. $\mathrm{Pd}_{43} \mathrm{Ni}_{10} \mathrm{Cu}_{27} \mathrm{P}_{20}$ with hydrated $\mathrm{B}_{2} \mathrm{O}_{3}$ agent was considered for prefoam production using this method. Hydrated $\mathrm{B}_{2} \mathrm{O}_{3}$ releases water vapor at temperatures above the liquidus temperature of the alloy, ${ }^{6}$ and does not reduce its glass forming ability. In fact it was observed that $\mathrm{B}_{2} \mathrm{O}_{3}$ could even improve the glass forming ability of this alloy. ${ }^{12,13}$

In the context of this work, $\mathrm{Pd}_{43} \mathrm{Ni}_{10} \mathrm{Cu}_{27} \mathrm{P}_{20}$ was mixed with $50 \mathrm{vol} \%$ of $\mathrm{B}_{2} \mathrm{O}_{3}$ and was processed in a quartz tube at $1170 \mathrm{~K}$ for 5 min under argon atmosphere at 2 bar pressure. After being processed, the prefoam was water quenched to its amorphous state. $\mathrm{The} \mathrm{Pd}_{43} \mathrm{Ni}_{10} \mathrm{Cu}_{27} \mathrm{P}_{20}$ prefoam produced is shown in Fig. 7. The prefoam consists of 15 vol \% bubbles 


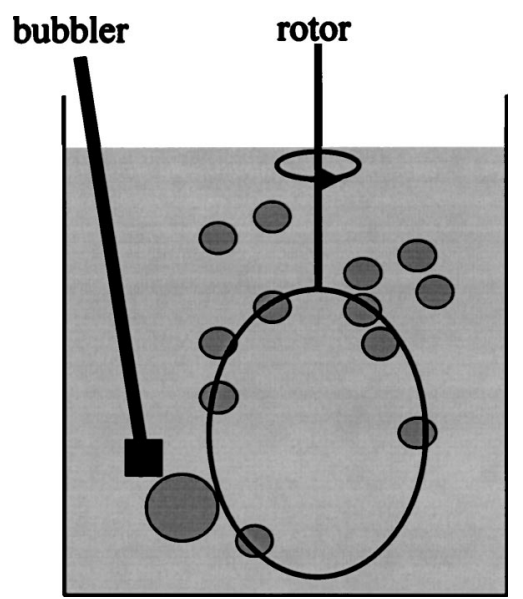

FIG. 8. Schematic drawing of the eggbeater constructed for the mechanical air-entrapment method. The setup comprises a molybdenum brush of $3 \mathrm{~cm}$ diameter spinning at speeds of up to $2500 \mathrm{rpm}$.

homogeneously distributed throughout the sample having an average size of $70 \mu \mathrm{m}$. Its amorphous nature was confirmed by thermal analysis.

The alternative prefoaming technique is to mechanically create bubbles in the liquid by air entrapment. In the mechanical air entrapment technique, bubbles are created as a consequence of induced liquid-gas instabilities, known as Rayleigh-Taylor instabilities. Such instabilities are often quantified by a dimensionless scaling number called the Weber number. The Weber number scales inertia forces to surface tension forces. It is defined as $\mathrm{We}=\rho u^{2} R / \sigma$, where $u$ is the relative velocity between liquid and brush and $\sigma$ is the liquid-gas surface tension. When We $>1$ inertia forces exceed interfacial tension forces and consequently interfacial instabilities are generated. Such instabilities develop at existing liquid-gas interface, and contribute to interface break-up such that small bubbles are generated. The Weber number can be employed to calculate the size of bubbles that can be created by assuming that bubbles can be broken up when We $>1$. Using typical values for density and surface tension as $\rho=6500 \mathrm{~kg} / \mathrm{m}^{3}$ and $\sigma=1 \mathrm{~N} / \mathrm{m}$ and a relative velocity of $u=3 \mathrm{~m} / \mathrm{s}$, the smallest bubble radius that can be broken up based on the above considerations is $\sim 20 \mu \mathrm{m}$.

With a kitchen eggbeater as a role model, a setup was built as shown schematically in Fig. 8. The setup comprises a molybdenum whisk of $3 \mathrm{~cm}$ diameter that is spinning at speeds of up to $2500 \mathrm{rpm}$. This results in relative velocities between liquid and brush of up to $3 \mathrm{~m} / \mathrm{s}$. The liquid sample is processed in a graphite crucible that is inductively heated. Small bubbles are created by breaking up large bubbles originated by either entrapping gas through the surface or by releasing gas through a bubbler positioned underneath the whisk. In the mechanical air entrapment technique, relatively high material quantities are required due to the large size of crucible needed in this process. Therefore $\mathrm{Zr}_{58.5} \mathrm{Nb}_{2.8} \mathrm{Cu}_{15.6} \mathrm{Ni}_{12.8} \mathrm{Al}_{10.3}$ alloy was instead considered in this prefoam technique due to its relatively low cost.

Figure 9(a) shows a $\mathrm{Zr}_{58.5} \mathrm{Nb}_{2.8} \mathrm{Cu}_{15.6} \mathrm{Ni}_{12.8} \mathrm{Al}_{10.3}$ prefoam synthesized by the mechanical air entrapment method. The quenched prefoam consists of 10 vol \% bubbles with an av-
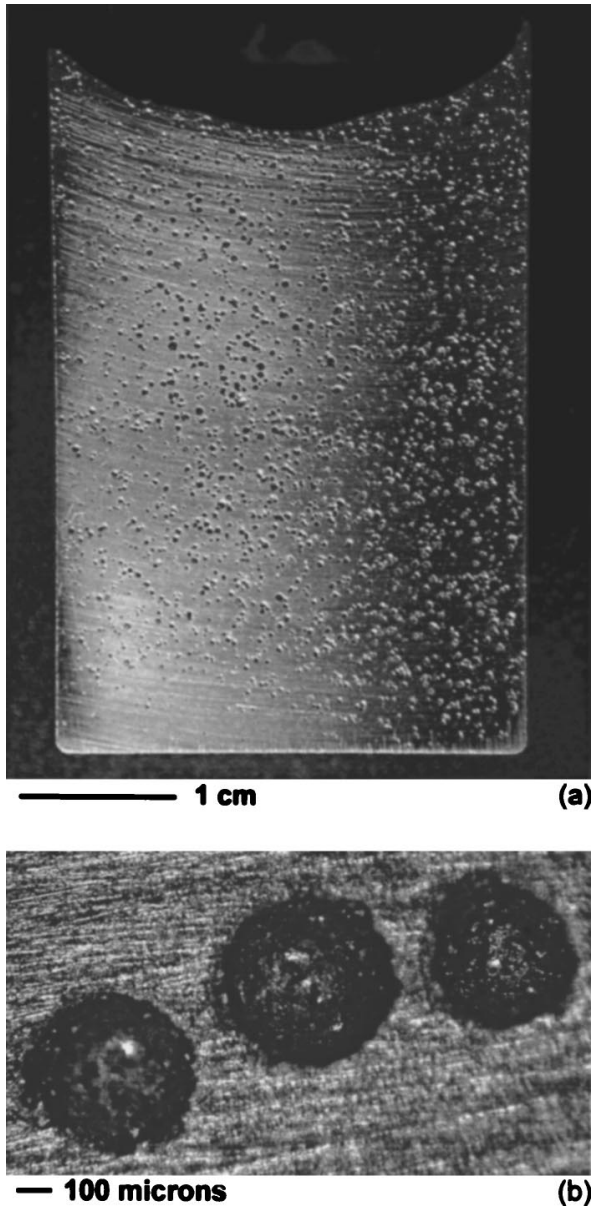

FIG. 9. (a) $\mathrm{Zr}_{58.5} \mathrm{Nb}_{2.8} \mathrm{Cu}_{15.6} \mathrm{Ni}_{12.8} \mathrm{Al}_{10.3}$ prefoam synthesized by the mechanical air entrapment method. It consists of $10 \mathrm{vol} \%$ bubbles with an average size of $250 \mu \mathrm{m}$. (b) Magnified view. The bubble size distribution appears fairly narrow.

erage size of $250 \mu \mathrm{m}$. The spatial distribution of bubbles appears to be fairly uniform, which implies that sedimentation was negligible during processing. Furthermore the size distribution of bubbles appears fairly narrow [see magnified view in Fig. 9(b)]. It can therefore be concluded that prefoams exhibiting adequate homogeneity can be produced by the air entrapment technique.

\section{B. Foam expansion}

In the foaming stage, prefoams were heated into the supercooled liquid region by means of a compact-resistance heater, or alternatively by an rf coil. The samples were then annealed at a given temperature in the supercooled region and foam expansion was activated by reducing the ambient pressure. The ambient pressures imposed in the foaming stage were of the order of $10^{-3}$ mbar.

Figure 10 (a) shows a $\mathrm{Pd}_{43} \mathrm{Ni}_{10} \mathrm{Cu}_{27} \mathrm{P}_{20}$ foam exhibiting $85 \%$ bubble volume fraction. The foam was processed by rf-coil heating with a heating rate of $40 \mathrm{~K} / \mathrm{min}$. Figure 10 (a) therefore demonstrates that production of low-density foam is possible using this synthesis method. The magnified view of this foam is shown in Fig. 10(b). This figure depicts that bubbles deviate from their spherical geometry at such low 

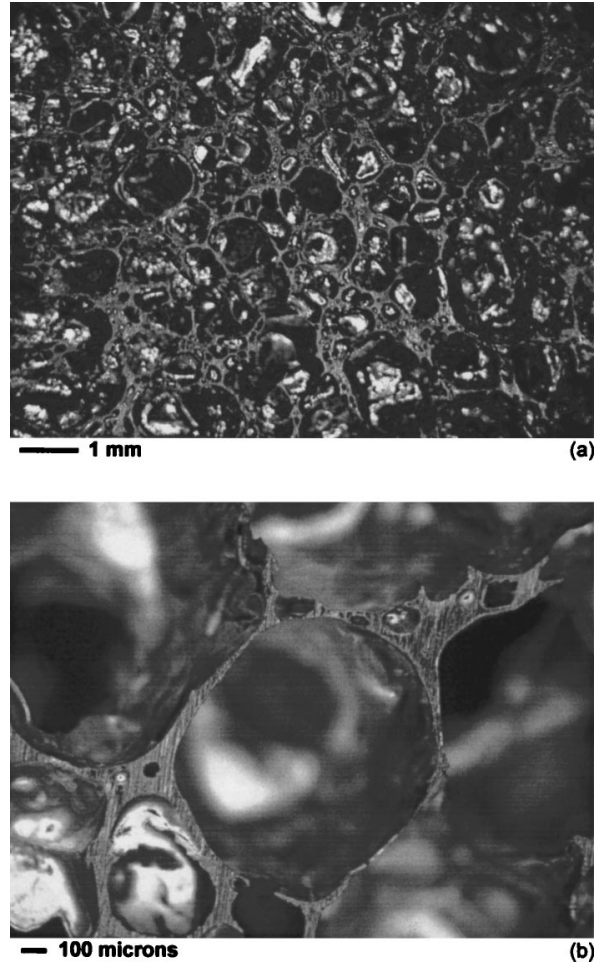

FIG. 10. (a) $\mathrm{Pd}_{43} \mathrm{Ni}_{10} \mathrm{Cu}_{27} \mathrm{P}_{20}$ foam expanded for 6 min by means of rf-coil heating. The foam exhibits a bubble volume fraction of $85 \%$. (b) Magnified view. Bubbles deviate from spherical symmetry, however, bubble merging appears to be restrained by the interconnecting films.

densities, however, bubble merging appears to be restrained by the interconnecting films. This points to a self-stabilizing mechanism between bubbles in $\mathrm{BMG}$ foams.

In Fig. 11, a $\mathrm{Pd}_{43} \mathrm{Ni}_{10} \mathrm{Cu}_{27} \mathrm{P}_{20}$ prefoam is shown together with the corresponding expanded foam. This figure illustrates bulk expansion of the sample, which is a consequence of the foaming process employed in this synthesis method. The prefoam consisted of $10 \mathrm{vol} \%$ of bubbles and was processed by the gas-releasing agent method under 3 bar pressure. The expanded foam exhibits a bubble volume fraction of $75 \%$. The amorphous nature of the foam was verified by thermal analysis.

Figure 12 shows a $\mathrm{Pd}_{43} \mathrm{Ni}_{10} \mathrm{Cu}_{27} \mathrm{P}_{20}$ prefoam along with two stages of expanded foam. The prefoam [Fig. 12(a)] consisted of $10 \mathrm{vol} \%$ of bubbles and was processed by the gasreleasing agent method under 3 bar pressure. Foam processing was performed by means of a compact-resistance heater. The processing time for the first stage [Fig. 12(b)] was $7.5 \mathrm{~min}$, and for the second stage [Fig. 12(c)] was $30 \mathrm{~min}$. The bubble volume fraction in the first expansion stage was

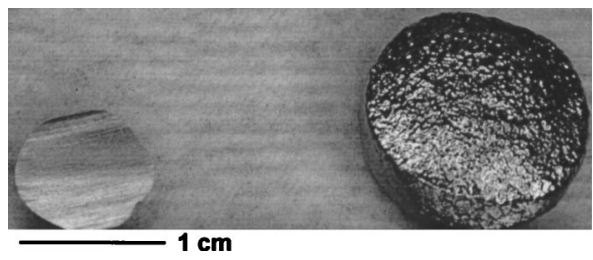

FIG. 11. Size comparison of $\mathrm{Pd}_{43} \mathrm{Ni}_{10} \mathrm{Cu}_{27} \mathrm{P}_{20}$ prefoam (left) and expanded foam (right) by rf-coil. The bubble volume fraction of the prefoam is $10 \%$, while that of the final foam is $75 \%$.

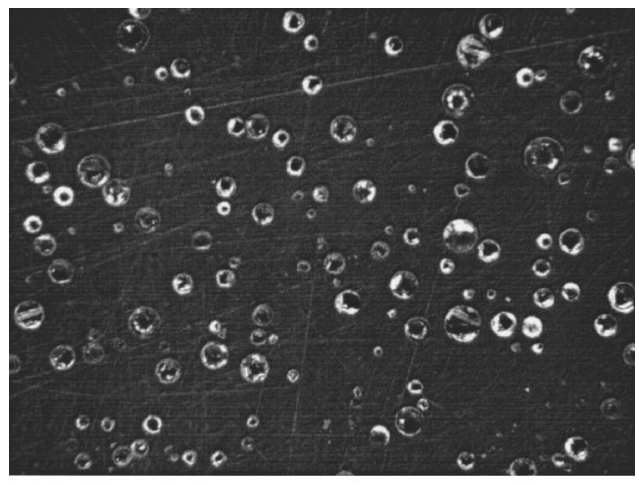

-100 microns

(a)

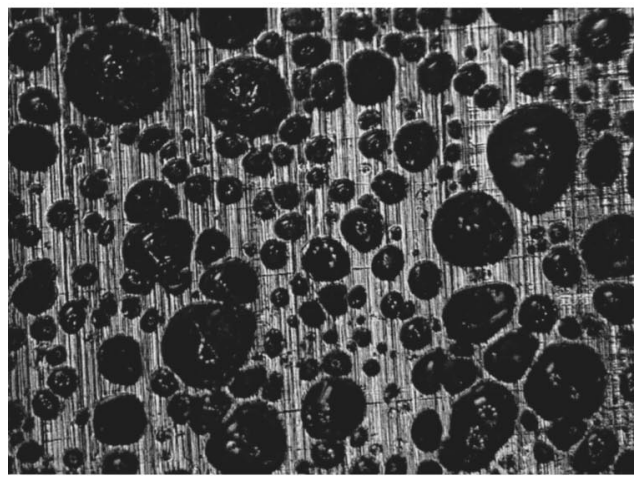

-100 microns

(b)

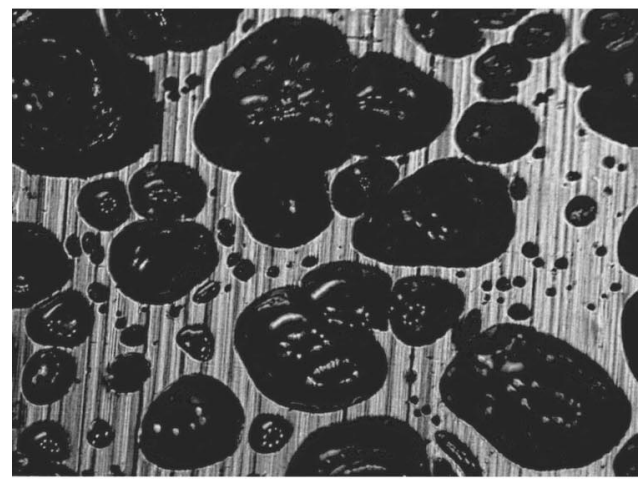

-100 microns

(c)

FIG. 12. Microstructure growth evolution of $\mathrm{Pd}_{43} \mathrm{Ni}_{10} \mathrm{Cu}_{27} \mathrm{P}_{20}$ liquid processed by compact-resistance heating. (a) Prefoam (10 vol \% bubbles). (b) Processed for $7.5 \mathrm{~min}$ (50 vol \% bubbles). (c) Processed for $30 \mathrm{~min}$ (65 vol \% bubbles).

$50 \%$, while in the second was $65 \%$. In Fig. 12 it is clearly demonstrated that by the proposed synthesis method it is possible to expand the foam in stages, which allows controllability over the final foam density.

The foam products developed in this study by the proposed synthesis method do not exhibit any observable density gradients attributed to bubble sedimentation. However, non-uniformities in the foam morphologies appear as a consequence of temperature gradients during foaming. These findings therefore validate the discussion in Sec. II.

\section{CONCLUSIONS}

A foam-synthesis method that utilizes the thermodynamic stability and thermoplastic formability of the super- 
cooled liquid state is introduced. In the proposed method, creation and expansion of bubbles becomes decoupled, as follows.

(1) In the prefoam processing step, a large number of small bubbles is created in the equilibrium liquid under pressure.

(2) In the prefoam quenching step, the bubbly liquid is quenched to its amorphous state.

(3) In the foam expansion step, the sample is reheated to the supercooled liquid region and the ambient pressure is substantially reduced with respect to the pressure used in the prefoaming step.

In the proposed synthesis method, cooling rate constraints on vitrification are relaxed and hence amorphous metallic foams can be produced in low densities and dimensions that are not limited to the critical casting thickness.

The expansion kinetics are evaluated by means of a dynamic model, which is utilized to assess whether adequate expansion is possible before crystallization sets in. The model suggests that expansion in the deeply undercooled liquid is feasible, as a processing time window exists between expansion and crystallization even when considering the most conservative bubble-growth kinetic conditions.

Within the proposed synthesis method, bulk amorphous metallic foams characterized by bubble volume fractions of as high as $85 \%$ are successfully produced. The foam products do not exhibit any observable density gradients attributed to bubble sedimentation, however, nonuniformities in the foam morphologies appear as a consequence of temperature gradients during foaming. It was also demonstrated in this study that foaming could be performed in a controlled manner such that desired foam densities can be accomplished.

\section{ACKNOWLEDGMENTS}

This work was supported by Liquidmetal Technology and NASA Marshall Space Flight Center. Dr. William F. Kaukler of the Center for Microgravity and Materials Research at The University of Alabama in Huntsville is acknowledged for engineering the compact-resistance heater. The authors express their gratitude to Kristen Virdone and Joan Christodoulou for technical assistance.

${ }^{1}$ J. Banhart, Prog. Mater. Sci. 46, 559 (2002).

${ }^{2}$ A. E. Evans, J. W. Hutchinson, and M. F. Ashby, Prog. Mater. Sci. 43, 171 (1999).

${ }^{3}$ T. Iida and R. I. L. Guthrie, The Physical Properties of Liquid Metals (Clarendon, Oxford, 1988).

${ }^{4}$ A. Masuhr, T. A. Waniuk, R. Busch, and W. L. Johnson, Phys. Rev. Lett. 82, 2290 (1999).

${ }^{5}$ T. Wubben, H. Stanzick, J. Banhart, and S. Odenbach, J. Phys.: Condens. Matter 15, S427 (2003).

${ }^{6}$ J. Schroers, C. Veazey, and W. L. Johnson, Appl. Phys. Lett. 82, 370 (2003).

${ }^{7}$ G. J. Fan, H.-J. Fecht, and E. J. Lavernia, Appl. Phys. Lett. 84, 487 (2004).

${ }^{8} \mathrm{H}$. Poritsky, Proceedings of the First U.S. National Congress Appl. Mech. (ASME, 1952), p. 813.

${ }^{9}$ Lord Rayleigh, (J. W. Strutt),Philos. Mag. 34, 93 (1917).

${ }^{10}$ I. Egry, G. Lohofer, I. Seyhan, S. Schneider, and B. Feuerbacher, Int. J. Thermophys. 20, 1005 (1999).

${ }^{11}$ J. Schroers, Y. Wu, R. Busch, and W. L. Johnson, Acta Mater. 49, 2773 (2001).

${ }^{12}$ H. W. Kui, A. L. Greer, and D. Turnbull, Appl. Phys. Lett. 45, 615 (1984).

${ }^{13}$ J. Schroers, Y. Wu, and W. L. Johnson, Philos. Mag. A 82, 1207 (2002). 